\title{
Critical Current Density Enhancement in High Temperature Superconductors by Flux Pinning
}

\author{
Manzoor A. Malik ${ }^{1}$ \\ ${ }^{I}$ Department of Physics, University of Kashmir, Hazratbal - 190 006, Srinagar, India
}

\begin{abstract}
Technological applications of High Temperature Superconductors (HTSC) are limited by "Flux Creep", an undesired magnetic vortices movement, creating a pseudo-resistance that depresses both critical current density and critical field. For practical applications (like energy storage systems, current limiters, magnetic bearings, etc.), it is desired to have high values of Critical Current Density $\left(J_{C}\right)$ even at higher applied field. Flux Pinning is an established mechanism of enhancing $J_{C}$ in HTSC by Swift Heavy Ion irradiation. This paper explains as to how $J_{C}$ can be enhanced in HTSC and thereby make HTSC technologically more useful. Some recent works and our initiatives in this direction are also discussed.
\end{abstract}

Keywords - Superconductivity, High temperature, Transport properties, Critical Currents, Flux Pinning

\section{Introduction}

High Temperature Cuprate Oxide Superconductors are extreme type-II, characterized by very high values (100 Tesla or greater) of the upper critical magnetic field $\left(\mathrm{Hc}_{2}\right)$. Combined with the relatively high Transition Temperatures $\left(\mathrm{T}_{\mathrm{C}}\right)$, above the easily accessible liquid nitrogen, it opens up a multitude of potential applications including magnets of incredible strength, storage systems, current limiters etc. However, $\mathrm{H}_{\mathrm{C}}$ and $\mathrm{T}_{\mathrm{C}}$ are not the only critical parameters that can destroy superconductivity. Another important critical parameter is the critical current density $\left(\mathrm{J}_{\mathrm{C}}\right)$, which unfortunately is inherently low for the high temperature superconductors.

The low critical current density is largely a consequence of an undesired phenomenon called Flux Creep, the ease with which magnetic vortices can move. This motion of fluxons creates a pseudo-resistance which depresses both critical current density and critical field, thereby limiting the technological applications of High Temperature Superconductors.

However, there is a way to enhance the critical current density in HTSC by somehow arresting the movement of these fluxons. This is called Flux Pinning and can be achieved by introducing artificial defects in the crystalline structure of the superconductor (apart from those that occur naturally).

\section{ENHANCEMENT OF $\mathbf{J}_{\mathrm{C}}$ BY ION IRRADIATION}

Ion irradiation with controlled energy is a well-known technique to introduce defects in materials [1]. The effect of ion beams on materials depends on the ion energy, fluence, ion species and material temperature. Haugan et al. [2] have shown that high density pinning centres can be achieved in YBCO in the form of $\sim 8 \mathrm{~nm}$ nano particles which will increase $\mathrm{J}_{\mathrm{C}}$ by a factor of two or three at high magnetic field. $\mathrm{NdBa} 2 \mathrm{Cu} 3 \mathrm{O} 7-\delta$ (NBCO) thin films (2000 $\AA$ ) grown by excimer $\mathrm{KrF}$ laser, irradiated with $\mathrm{Au}+13(200 \mathrm{MeV})$ ions, show an almost four-fold increase in $\mathrm{J}_{\mathrm{C}}$ at the optimum ion dose, indicating a robust pinning of vortices [3]. An enhancement of $\mathrm{J}_{\mathrm{C}}(\mathrm{H})$ in La-2125 thin films has been reported [4] with increasing Ag+15 ion irradiation upto an ion fluence of $1 \times 10^{11}$ ions per $\mathrm{cm}^{2}$. Interestingly, the maximum enhancement in $\mathrm{J}_{\mathrm{C}}$ is observed to be five-fold higher $\left(1.6 \times 10^{7} \mathrm{~A} . \mathrm{cm}-2\right)$ compared to $\mathrm{J}_{\mathrm{C}}$ of pristine thin films. In their study on the effect of Ag15+ and Li3+ ion irradiation on superconducting $\mathrm{Tl} 2 \mathrm{Ca} 2 \mathrm{Ba} 2 \mathrm{Cu} 3 \mathrm{O} 10$ single crystals, M. R. Singh et al [5] reported enhancement in $\mathrm{J}_{\mathrm{C}}$. Mele et al [6] successfully introduced high-density linear defects as artificial pinning centers (APCs) of the quantized vortices into YBCO films. They observed that $\mathrm{J}_{\mathrm{C}}$ increased to $1.8 \times 10^{7} \mathrm{~A} / \mathrm{cm}^{2}$, which is 1.5 times higher than that of the pure YBCO film even if only $10 \%$ of the induced defects act as effective pinning centers. $R$ Biswal et al [7] have reported a decrease of $T_{C}$ at low fluencies on creation of point defect by swift heavy ion irradiation-induced low energy electrons in $\mathrm{YBa} 2 \mathrm{Cu} 3 \mathrm{O} 7-\delta$. Recently, Sung Hun Wee et al [8] have shown the improvement of flux pinning and $\mathrm{J}_{\mathrm{C}}$ performance over wide magnetic field ranges in $\mathrm{YBa}_{2} \mathrm{Cu}_{3} \mathrm{O}_{7}-\delta$. They found that RTO (RE3TaO7) addition resulted in an improvement of the critical current density, $\mathbf{J}_{\mathrm{C}}$. Similar increase of $\mathbf{J}_{\mathrm{C}}$ was reported by Haruta et al [9] on introduction of columnar defects as pinning centres in high- $\mathrm{T}_{\mathrm{C}}$ superconductors.

Large enhancements in the critical current density $\mathrm{J}_{\mathrm{C}}$ have been reported in single crystals of the high-temperature superconductor $\mathrm{Bi} 2 \mathrm{Sr} 2 \mathrm{Ca} 1 \mathrm{Cu} 2 \mathrm{O} 8$ by irradiation with high energy $\mathrm{Sn}$ ions [10]. Bugoslavsky et al studied the effect of proton irradiation in superconducting $\mathrm{MgB}_{2}$ single crystals [11] and observed that at high magnetic fields the irradiation of the crystal has a pronounced effect across the whole temperature range. 
Several other studies, many of them carried out at IUAC, New Delhi (Summarized in [1, 12]), have confirmed that SHI irradiation modifies the properties in several Superconducting systems. Some studies, however, show a flip side of the effects of SHI irradiation. For example, Sarkar et al [13] have reported that high-energy particle beam limits applications of HTSC as superconducting magnets and other devices.

\section{BZO As PINNING Centres}

Some recent studies have shown encouraging results for critical current density enhancement on doping various superconducting films with $\mathrm{BaZrO} 3$ (BZO) nanoparticles. The main reasons for using $\mathrm{BZO}$ as artificial pinning centres in YBCO are: (a) BZO has a high melting temperature with respect to $\mathrm{YBCO}$ and so the growth kinetics should be slow, leading to small particles, (b) large lattice mismatch (approx. 9\%), so strain between the phases could introduce defects for enhanced pinning. The pinning and structural properties, however, depend on the YBCO and BZO layer thicknesses, hence the structure should be optimised for desired thin film properties.

Ijaduola et al [14] studied $\mathrm{J}_{\mathrm{C}}$ for a set of $\mathrm{NdBa} 2 \mathrm{Cu} 3 \mathrm{O} 7$ (NdBCO) films doped with $\mathrm{BaZrO} 3$ (BZO) nanoparticles and found that current density in the doped samples increased by a factor of 2.4. Study of film growth of $\mathrm{BaZrO3}$-doped $\mathrm{YBa}_{2} \mathrm{Cu}_{3} \mathrm{O}_{7}$ by using fluorine-free metal-organic deposition by $\mathrm{Lu}$ et al [15] shows that critical current density $\left(\mathrm{J}_{\mathrm{C}}\right)$ and the pinning force are greatly enhanced in the BZO-doped sample at $77 \mathrm{~K}$ relative to pure YBCO films. Goyal et al [16] have achieved linear defects in YBCO by chemical doping with $\mathrm{BaZrO} 3$ resulting in substantial increase in $\mathrm{J}_{\mathrm{C}}$ and vortex pinning, but progressive depression of $\mathrm{T}_{\mathrm{C}}$. Several other studies have underlined the role of BZO doping in changing various properties of superconductors, in particular, enhancement of $\mathrm{J}_{\mathrm{C}}$.

\section{DISCUSSION}

For purposeful technological applications of high temperature superconductors, it is important to find ways for improving the critical current density. The main reason for using SHI irradiation for improving $\mathrm{J}_{\mathrm{C}}$ in superconductors is that it is known to introduce point and columnar defects which act as effective pinning centres thereby restricting the movement of fluxons which in turn results in a gain in $\mathrm{J}_{\mathrm{C}}$. Recent studies involving doping of BZO nanoparticles in superconducting films has opened a new front. As pointed out in the preceeding section, large lattice mismatch and high melting temperature with respect to YBCO make it ideal for enhancement of $\mathrm{J}_{\mathrm{C}}$, even without ion irradiation.

At IUAC (Inter University Accelerator Centre, New Delhi), effect of ion irradiation on several properties of superconductors have been conducted. These include the Critical Current Density, Transition Temperatures, Critical Magnetic Fields and other transport and magnetic properties of superconductors. BZO doping has not so far been studied at IUAC. We plan to optimise YBCO and BZO film thickness for best $\mathrm{J}_{\mathrm{C}}$. We also intend to study the effect of SHI irradiation on the upper critical magnetic field $\left(\mathrm{Hc}_{2}\right)$. Further, since the phase diagram of high temperature superconductors shows different regimes like antiferromagnetism, semiconduction, superconductivity, strongly correlated electrons and metallic properties when plotted as a function of the doping, we expect to gain some insight of the normal state of high temperature superconductors.

\section{REFERENCES}

[1] D. K. Avasthi and G K Mehta, Swift Heavy Ions for Materials Engineering and Nanostructuring, Springer, 2011.

[2] T. J. Haugan et al. Nature, 430, 867, 2004

[3] K. Sanghamitra et al, Supercond. Sci. Technol., 15, 324, 2002

[4] K. R. Mavani et al, Solid State Communications, 142, 462, 2007

[5] M. R. Singh et al., Nucl. Instru. and Meth., B 269, 11172011

[6] P. Mele et al., Physica C , 426, 1108, 2005

[7] R. Biswal et al, Supercond. Sci. Technol. 21, 085016, 2008

[8] S. H. Wee et al, J. of the American Ceramic Society, 95, 4, 1174, 2012

[9] M. Haruta et al, Physica C, 412, 511, 2004

[10] R. E. Thomson et al, Appl. Phys. Lett., 63, 614, 1993

[11] Y. Bugoslavsky, Nature, 411, 561, 2001

[12] R. Kumar et al, Journal of Applied Physics 97, 093526, 2005

[13] Sarkar et al Ind. J. Cryog., 12, 213, 2005

[14] A. O. Ijaduola et al, Supercond. Sci. Technol. 25, 045013, 2012

[15] Lu et al, Supercond. Sci. Technol. 25, 015011, 2012

[16] A. Goyal et al, Superconduct. Sci. Technol. 18, 11, 1533, 2005 